\title{
Avaliação de indicadores de qualidade em hemodiálise
}

Evaluation of quality-of-care indicators in hemodialysis

\author{
${ }^{1}$ João Márcio Nunes de Alencar \\ ${ }^{2}$ Lucyana Bertoso de Vasconcelos Freire
}

1. Bacharel em Enfermagem pela Universidade Federal do Piauí - UFPI. Especialista em Nefrologia pela Faculdade Unyleya. Residência em Nefrologia pela ESCS/FEPECS.

2. Tutora do Programa de Residência em Enfermagem em Nefrologia da Secretaria de Saúde do Distrito Federal- SES/FEPECS. Mestre em Enfermagem pela Universidade de Brasília.

\section{Resumo}

Avaliar os indicadores de qualidade em hemodiálise em um Hospital Universitário e propor estratégias para melhoria dessas metas nas intervenções de saúde relacionadas à hemodiálise. Metodologia: Estudo descritivo e exploratório, retrospectivo, com abordagem quantitativa. Resultados e Discussão: Houve predomínio da faixa etária acima de 60 anos; solteiros, sexo masculino e grau de escolaridade fundamental; renda média entre 1 a 3 salários mínimos e beneficiários do INSS. Os indicadores Hemoglobina, Kt/v e paratormônio, foram os que mais apresentaram não conformidade; fósforo e albumina plasmática, estavam dentro dos valores preconizados; infecções de acessos vasculares, IAVC, IPCS, internações e mortalidade, apresentaram grupos com valores em não conformidade com as diretrizes preconizadas. Fístula arteriovenosa maturada, uso de cateter temporário por mais de 3 meses e pacientes aptos inscritos para Transplante, apresentaram índices abaixo do recomendado. Conclusão: O estudo acerca de indicadores permite visão da qualidade do serviço e oferece oportunidade de intervenções eficazes. Observou-se necessidade de mais estudos com a temática em outros contextos metodológicos e assistenciais.

Palavras-chave: Doença Renal Crônica, Hemodiálise, Indicadores de qualidade.

\begin{abstract}
The main objective of this work is to evaluate quality indicators in hemodialysis in a University Hospital and to propose strategies in order to improve indicators within health interventions related to hemodialysis. Method: Descriptive and exploratory retrospective research, with a quantitative approach. Results and Discussion: The cohort consisted on mainly individuals over 60 years old, single, male, with a middle school educational level, average income between 1 and 3 minimum wages, and social security beneficiaries. Indicators accessed were as follows: hemoglobin, $\mathrm{Kt} / \mathrm{v}$ and parathormone, were the ones with the lowest conformity rates; phosphorus and plasmatic albumin, were within conformity rates; vascular access infections, primary bloodstream infections, hospital stays and mortality, showed some groups with low conformity rates; matured arteriovenous fistula, use of temporary catheter over 3 months and patients eligible and enrolled for transplantation, showed rates below recommended. Conclusion: The evaluation of hemodialysis quality-of-care indicators allows a broad overview on the quality of the provided service, therefore offering the opportunity to provide effective interventions. It is suggested that more studies on quality-of-care indicators should be conducted to determine main indicators common to different contexts and cohorts.
\end{abstract}

Keywords: Chronic Kidney Disease, Hemodialysis, Quality indicators, Quality-of-care 


\section{Introdução}

A Doença Renal Crônica (DRC) consiste em uma lesão renal com perda progressiva e irreversível da função dos rins ${ }^{1}$. Em sua fase mais avançada, os rins não conseguem manter a normalidade interna do paciente, que necessita ser submetido a alguma terapia de substituição renal $(\mathrm{TRS})^{1}$.

Estudos demonstram as altas taxas de incidência da doença, principalmente relacionada ao Diabetes Mellitus (DM) ${ }^{1}$. O número de pessoas com DRC cresce a cada ano e os dados da Sociedade Brasileira de Nefrologia (SBN), de $2017^{2}$, apontam que 126.583 pacientes realizam alguma TRS no Brasil, sendo que 93,1\% se encontra em hemodiálise (HD), apenas 6,9\% em diálise peritoneal (DP) e 31.226 (24\%) estavam em fila de espera de transplante renal (TX) ${ }^{2}$.

A DRC é caracterizada atualmente como um problema de saúde pública devido ao elevado número de pacientes que surgem anualmente, e, com isso, a necessidade constante de vagas para TRS. A maior parte desses pacientes chegam aos serviços de saúde nos estágios finais da doença, necessitando com urgência de alguma modalidade terapêutica. O Ministério da Saúde vem desenvolvendo diretrizes, metodologias e instrumentos para apoiar as equipes de saúde a prevenir, reduzir as complicações, tratar e recuperar a saúde desses pacientes, visando qualificar o cuidado integral ${ }^{1}$.

Alguns órgãos nacionais e internacionais, como a SBN ${ }^{2}$, o National Kidney Fundation $(\mathrm{NKF})^{3}$ e o painel Europeu de diálise - Dialysis Outcomes And Practice Patterns Study $(\text { DOPPS })^{4}$, propuseram diretrizes para avaliar o cuidado holístico aos pacientes em hemodiálise. As estratégias surgiram com a crescente necessidade de melhoria dos modelos organizacionais que resultem em serviços qualificados nos centros de diálise, atrelados a baixo custo e menor índice de erros ${ }^{5}$. 
Até meados de 2014, não havia obrigatoriedade de mensurações dos indicadores de qualidade na prática clínica e somente alguns, como o clearance de ureia $-\mathrm{Kt} / \mathrm{v}-$, eram medidos. Contudo, foi implementada a portaria número 389 de 13 de março de $2014^{6}$, da Agência Nacional de Vigilância Sanitária - ANVISA, que foi recentemente atualizada por meio da portaria $\mathrm{n}^{\mathrm{o}} 1675$ de 07 de junho de $2018^{7}$. Criou-se uma lista de indicadores prescritos, a serem mensurados e verificados como exigência aos serviços de diálise para avaliar a qualidade da assistência oferecida pelas unidades de saúde ${ }^{8}$.

A curva ascendente de pacientes ingressos em hemodiálise a cada ano exige a elaboração de estratégias que visem garantir que as modalidades de substituição da função renal sejam ofertadas no melhor padrão de qualidade. Nesse sentido, o foco de atuação das equipes deve ser a superação dessas barreiras por meio da utilização dos recursos disponíveis, como a mensuração e acompanhamento dos indicadores ${ }^{5}$.

Essas característica da doença e do tratamento na atual conjuntura do Brasil configuram uma problemática na assistência ao paciente com DRC no Brasil, empecilho presente de forma frequente quando se deseja avaliar o quão eficaz e eficiente está sendo o tratamento ofertado aos pacientes com $\mathrm{DRC}^{5,9}$.

A experiência do autor como enfermeiro em setor de hemodiálise trouxe vários questionamentos sobre o assunto. No desenvolvimento de atividades como profissional de enfermagem residente em Nefrologia, observou que a dinâmica do serviço, as especificidades do setor, as novas adequações da equipe de enfermagem relativas às contratações recentes e o grande número de pacientes parecem afetar diretamente a assistência, e apresentam-se como desafios na utilização dos indicadores.

Apesar de algumas taxas e índices serem verificados e registrados, a percepção é de que ainda há muito a avançar nesse aspecto. A inquietude em como e quais os meios para modificar essa realidade foi o ponto de partida para a realização desse estudo. 
Intercorrências acontecem diariamente na hemodiálise. Essas intercorrências podem ou não gerar graves consequências. Contudo, uma lista de indicadores, recentemente atualizada na portaria $n^{\circ} 1675$ de 16 de junho de $2018^{7}$, traz consigo cuidados necessários a uma assistência pautada na mais alta qualidade, que se seguida rigorosamente eleva os cuidados com os pacientes.

Diante do exposto, essa pesquisa teve por objetivo avaliar os indicadores de qualidade em hemodiálise em um Hospital Universitário do Distrito Federal e propor estratégias para melhoria dessas metas nas intervenções de saúde relacionadas à hemodiálise.

\section{Métodos}

Estudo descritivo e exploratório, retrospectivo, de janeiro a dezembro de 2018, com abordagem quantitativa. Todas essas formas de estudo descrevem algo de natureza genuína, sem acrescentar variáveis externas ${ }^{11}$.

Tornar o leitor familiarizado ao tema, além de proporcionar um fácil entendimento do assunto e, sempre que possível, construir hipóteses: a finalidade da pesquisa exploratória ${ }^{12}$. Estipula-se um determinado período em que se deseja coletar os dados, com a consequente seleção dos participantes da pesquisa, a partir de documentos notadamente autênticos ${ }^{13}$.

A pesquisa quantitativa refere-se a números de qualquer natureza, envolve a coleta sistêmica de informações em forma numérica, rotineiramente seguindo um determinado controle para posterior análise dessas informações utilizando de procedimentos estatísticos, a serem demonstrados de muitas maneiras, através de, por exemplo, gráficos em pontos, em pizza, em relevo, tabelas, quadros ou simplesmente por extenso ${ }^{14}$.

O estudo foi desenvolvido no Centro de Diálise (CD) do Hospital Universitário de Brasília - HUB. 
A população do estudo foi composta pelos pacientes crônicos cadastrados no CD do HUB que utilizam de hemodiálise como terapia de substituição renal. A amostra total de 28 pacientes, consiste naqueles que estavam no programa no período que abrange janeiro de 2018 a dezembro de 2018 .

Foram incluídos na pesquisa os pacientes renais crônicos em HD que realizaram sessão três vezes por semana no período de janeiro de 2018 a dezembro de 2018, de ambos os gêneros e acima de 18 anos, cujas variáveis estudadas estivessem registradas em prontuário físico e/ou eletrônico. Delimitou-se os seguintes critérios de exclusão: pacientes com prontuários incompletos, rasurados, com informações duplicadas ou que se negaram a participar da pesquisa em qualquer momento da coleta de informações.

A coleta dos dados ocorreu nos meses de novembro e dezembro de 2019. Os dados desse estudo foram obtidos por meio de consulta aos prontuários eletrônicos, físicos, resultados de exames, formulários, registros de enfermagem e médicos, anotações e quaisquer documentos que representem e se encaixem nas variáveis da pesquisa, todos armazenados no setor.

Utilizamos de questionário próprio, constituído de perguntas estruturadas que avaliam os indicadores de qualidade em hemodiálise citados na RDC 389 de 13 de março de $2014{ }^{6}$ e portaria $\mathrm{n}^{\mathrm{o}} 1675$ de 07 de junho de $2018^{7}$ e adaptados à realidade do setor em questão.

As metas dos indicadores foram pré-estabelecidas e extraídas das resoluções e portarias que regulamentam o serviço de diálise brasileiro ${ }^{7-9}$, especificadas pelo quadro 1. 
Quadro 1: Avaliação de Indicadores de qualidade em Hemodiálise

\begin{tabular}{|c|c|c|c|c|}
\hline Frequência & Indicador & & Meta & Cálculo \\
\hline \multirow[t]{5}{*}{ Trimestral: } & $\begin{array}{l}- \text { Proporção de } \\
\text { pacientes com Hb }>10 \\
\text { g/dl e < } 12,0 \mathrm{~g} / \mathrm{dl} \mathrm{em} \\
\text { diálise; }\end{array}$ & & $80 \%$ & $\begin{array}{l}\quad \mathrm{N}^{\mathrm{o}} \text { de pacientes em } \\
\text { diálise com } \mathrm{Hb}>10 \text { e }<12 \\
\mathrm{~g} / \mathrm{dl} / \mathrm{N}^{\mathrm{o}} \text { total de pacientes em } \\
\text { diálise X 101; }\end{array}$ \\
\hline & $\begin{array}{l}\quad \text { Proporção de } \\
\text { pacientes em diálise com } \\
\text { Fósforo }(\mathrm{P})>3,5 \text { e }<5,5 \\
\mathrm{mg} / \mathrm{dl} ;\end{array}$ & & $50 \%$ & $\begin{array}{l}\quad \mathrm{N}^{\mathrm{o}} \text { de pacientes com } \mathrm{P} \\
>3,5 \text { e }<5,5 \mathrm{mg} / \mathrm{dl} / \mathrm{N}^{\circ} \text { total de } \\
\text { pacientes em diálise X } 101 ;\end{array}$ \\
\hline & $\begin{array}{l}\quad \text { Proporção de } \\
\text { pacientes com Clearence } \\
\text { de ureia }=>1.3 ;\end{array}$ & & $80 \%$ & $\begin{array}{l}\mathrm{N}^{\mathrm{o}} \text { pacientes com } \mathrm{Kt} / \mathrm{v} \\
\text { maior que } 1,3 / \mathrm{N}^{\circ} \text { total de } \\
\text { pacientes em tratamento de } \\
\text { HD X } 100\end{array}$ \\
\hline & $\begin{array}{l}\quad \text { Proporção de } \\
\text { pacientes com valor de } \\
\text { PTH > } 600 \mathrm{pg} / \mathrm{ml} ;\end{array}$ & $\%$ & $<20$ & $\begin{array}{l}\quad \mathrm{N}^{\circ} \text { de pacientes com } \\
\text { PTH> } 600 \mathrm{pg} / \mathrm{ml} / \mathrm{N}^{\circ} \text { total de } \\
\text { pacientes em tratamento } \mathrm{X} \\
100\end{array}$ \\
\hline & $\begin{array}{l}\text { Proporção de } \\
\text { pacientes com valores de } \\
\text { albumina sérica }<3\end{array}$ & & $20 \%$ & $\begin{array}{l}\mathrm{N}^{\mathrm{o}} \text { pacientes com } \mathrm{Ab} \\
\text { menor que } 3,0 \mathrm{~g} / \mathrm{dl} / \mathrm{N}^{\circ} \text { total de } \\
\text { pacientes em tratamento } \mathrm{X} \\
100\end{array}$ \\
\hline \multirow[t]{3}{*}{ Mensal: } & 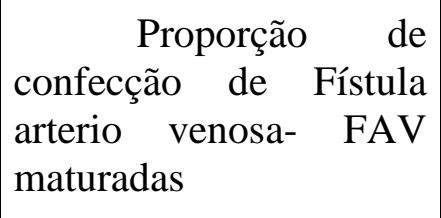 & & $70 \%$ & $\begin{array}{l}\mathrm{N}^{\circ} \text { pacientes com FAV } \\
\text { maturadas/N de pacientes } \\
\text { com FAV confeccionadas X } \\
100\end{array}$ \\
\hline & $\begin{array}{l}\text { Incidência de } \\
\text { Infecção de acesso } \\
\text { venoso central }\end{array}$ & $\%$ & $<10$ & $\begin{array}{l}\quad \mathrm{N}^{\mathrm{o}} \text { pacientes com } \\
\text { IAVC } / \mathrm{N}^{\mathrm{o}} \text { total de pacientes } \\
\text { em tratamento X } 100\end{array}$ \\
\hline & $\begin{array}{l}\text { Incidência de } \\
\text { pacientes com infecção } \\
\text { primária de corrente } \\
\text { sanguínea; }\end{array}$ & $\%$ & $<10$ & $\begin{array}{l}\quad \mathrm{N}^{\circ} \text { pacientes com } \\
\text { IPCS } / \mathrm{N}^{\circ} \text { total de pacientes em } \\
\text { tratamento } \mathrm{X} 100\end{array}$ \\
\hline
\end{tabular}




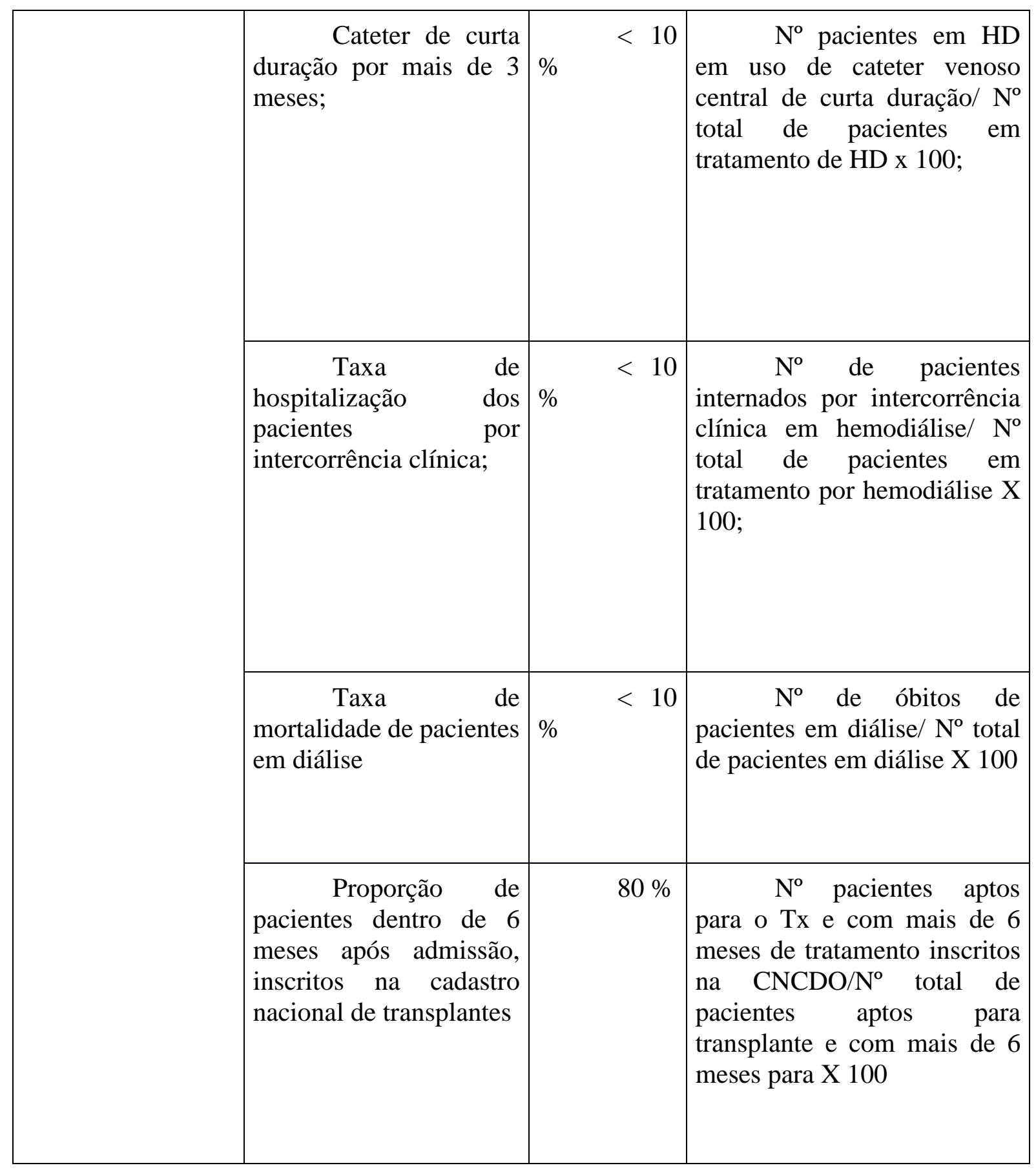

A amostra final de participantes foi agrupada por tempo em meses de diálise, devido à heterogeneidade dos indicadores. Para facilitar o entendimento, os grupos foram classificados considerando o tempo em meses completos na diálise, assim denominados: Grupo $1(<3$ meses), Grupo 2 ( $>3$ e $<6$ meses), Grupo 3 ( $>6<9$ meses), Grupo 4 ( $>9-12$ meses) (Quadro 2). 
Os números sumarizados do questionário foram adicionados a uma planilha de Microsoft Excel 2015 para posterior cálculo dos escores, média e desvio padrão. A análise dos dados foi feita por meio de estatística descritiva e quantitativa, observando quais foram os indicadores de qualidade que mais apresentaram influência e impacto, seja positivo ou negativo, na qualidade da hemodiálise.

A pesquisa não envolveu seres humanos, somente arquivos de prontuários físicos e eletrônicos, dispensando a aplicação de TCLE, justificativas para tal informadas na plataforma Brasil. Aprovado pelo Comitê de Ética em Pesquisa da Fundação em Ensino e Pesquisa FEPECS, número de parecer 3.654.464

\section{Resultados e Discussões}

A pesquisa teve amostra total de 28 pacientes (tabela 01), com predomínio do sexo masculino $16(57,1 \%)$. Resultados semelhantes foram encontrados no estudo de $\operatorname{Veras}^{5}$, no Censo da SBN referente ao ano de 2017 Thomé $^{16}$, ambos com $58 \%$, Melo ${ }^{17}$, com 59\%, e no estudo internacional DOPPS ${ }^{4}$, com prevalência de $60 \%$ de pacientes do sexo masculino.

Indivíduos do sexo masculino cuidam menos da saúde, quando em comparação com as mulheres, expondo-se mais às situações de risco, como hipertensão arterial e diabetes mellitus 17. Os homens se tornam mais suscetíveis a doenças renais, uma vez que julgam-se menos vulneráveis e procuram menos os serviços de saúde ${ }^{5}$.

No estudo houve predomínio de pacientes idosos, prevalecendo aqueles com mais de 60 anos (42,9\%); a média das somas das idades resultou em 50,71 anos (tabela 01). Dados semelhantes foram encontrados no Censo de 2017, com maior número de paciente entre 45 e 64 anos $(42,6 \%)^{16}$. A média em anos no estudo de Veras ${ }^{5}$ foi de 55,54 anos. No estudo de Cavalcanti ${ }^{10}$, a média foi de 50,4 anos, análogo aos valores encontrados na nossa pesquisa. 
De acordo com dados colhidos, $16(57,1 \%)$ (tabela 01) pacientes possuíam grau de escolaridade fundamental, equivalente a 4 - 7 anos de estudos. Corroborando com o estudo de Cavalcanti ${ }^{10}$, em que encontrou uma taxa de $50 \%$ dos participantes da pesquisa com grau de escolaridade no ensino fundamental. Nerbas ${ }^{18}$ também encontrou prevalência de ensino fundamental - pelo menos metade da amostra total tinha entre 4- 7 anos de estudo. Divergindo dos dados anteriores, Veras ${ }^{5}$ teve taxa de analfabetismo funcional em alta: $33 \%$ dos pesquisados possuíam menos que 4 anos de estudos.

Quanto ao estado civil dos participantes, maior número deles estava em uma união estável (35,7\%) (tabela 01). Dados semelhantes foram encontrados no estudo de Cavalcanti ${ }^{10}$ e Pereira e Leite ${ }^{19}$, onde predominou união estável, $56,1 \%$ e 52,7\%, respectivamente.

Os pacientes acompanhados no estudo apresentaram renda média de 1 a 3 salários mínimos, representando $81,2 \%$ (tabela 01) do total da amostra. Resultado equivalente foi encontrado no estudo de Veras ${ }^{5}$ (73\%). No estudo nacional dos pesquisadores Araújo-Ferreira ${ }^{20}$, onde maior parte dispunha de uma renda média entre 1 a 2 salários mínimos, 60,8\% eram beneficiários do INSS, 9,8\% desempregados e 29,4\% tinham outra ocupação.

Dados de censo da SBN de 2017, mostram que mais de $82 \%$ do tratamento dialítico no país é pago pelo SUS, levando a uma sobrecarga de gastos, e às vezes, falta de vagas dialíticas 16.

Dos participantes, 67,9\% eram beneficiários de seguridade social, 10,7\% estavam ativos, exerciam alguma atividade remunerada com carteira assinada e 21,4\% eram autônomos (tabela 01). Esses dados corroboram com o estudo de Pereira e Leite ${ }^{19}$, onde $92,2 \%$ eram beneficiários do INSS e somente 4,7\% estavam ativos no mercado. Os baixos valores de renda refletem o percentual de plano de saúde na amostra: 57\% não possuía plano, e, destes, somente 17,4\% tinha seu tratamento custeado pelos planos privados de saúde. 
Tabela 01- Distribuição dos pacientes renais crônicos segundo as características sociodemográficas, HUB, Brasília, 2020.

\begin{tabular}{l|ll} 
Idades & Soma & Média \\
\hline & 1420 & 50,71 \\
$\leq 29$ anos & Quant. & $\%$ \\
\cline { 2 - 2 } $30-39$ anos & 2 & $7,1 \%$ \\
$40-49$ anos & 4 & $14,3 \%$ \\
$50-59$ anos & 4 & $14,3 \%$ \\
$\geq 60$ anos & 6 & $21,4 \%$ \\
Total & 12 & $42,9 \%$ \\
& 28 & $100,0 \%$
\end{tabular}

\begin{tabular}{l|ll} 
Sexo & Quant. & Percentual \\
\hline Masculino & 16 & $57,1 \%$ \\
Feminino & 11 & $39,3 \%$ \\
Transexual & 01 & $3,57 \%$ \\
Total & 28 & $100 \%$
\end{tabular}

\begin{tabular}{l|lc} 
Escolaridade & Quant. & Percentual \\
\hline Não alfabetizado & 4 & $14,3 \%$
\end{tabular}


Ensino Fundamental

Ensino médio

Ensino Superior

Sem relatos

Total
16

4

1

3

28
$57,1 \%$

$14,3 \%$

$3,6 \%$

$10,7 \%$

$100,0 \%$

\begin{tabular}{l|ll} 
Estado Civil & Quant. & Percentual \\
\hline Sem companheiro(a) & 13 & $46,4 \%$ \\
Casado/união estável & 10 & $35,7 \%$ \\
Divorciado(a) & 5 & $17,9 \%$ \\
Total & 28 & $100,0 \%$
\end{tabular}

\begin{tabular}{l|ll} 
Renda & Quant. & Percentual \\
\hline Até 01 salário mínimo & 4 & $14,3 \%$ \\
01 a 03 salários mínimos & 23 & $82,1 \%$ \\
03 a 05 salários mínimos & 0 & $0,0 \%$ \\
Mais de 05 salários mínimos & 1 & $3,6 \%$ \\
Total & 28 & $100,0 \%$
\end{tabular}

\begin{tabular}{l|lc} 
Ocupação & Quant. & Percentual \\
\hline Carteira assinada & 3 & $10,7 \%$
\end{tabular}


Desempregado

Beneficiário do INSS

Autônomo

Total
$0 \%$

$19 \quad 67,9 \%$

$6 \quad 21,4 \%$

$28 \quad 100,0 \%$

O indicador hemoglobina $(\mathrm{Hb})$ não atingiu meta em nenhum dos grupos do estudo, considerando os indicadores do Ministério da Saúde que recomendam meta de 80\%, a relatar: grupo $1(33,33 \%)$, grupo $2(56,25 \%)$, grupo $3(39,73 \%)$ e grupo $4(50,77)$ (tabela 2$)$, muitos desses estavam acima do valor padronizado $12 \mathrm{~g} / \mathrm{dl}$, configurando poliglobulia.

Dados semelhantes foram descrito por Veras ${ }^{5}$, onde $64 \%$ da amostra apresentaram níveis de hemoglobina menor que o ideal. Pereira e Leite ${ }^{19}$, obtiveram amostra com 53,1\% de pacientes com hemoglobina abaixo da meta. Valores mais expressivos foram vistos no estudo de Cavalcanti ${ }^{10}$, no qual $73,2 \%$ da amostra tinha $\mathrm{Hb}$ diminuída.

O difícil controle da anemia ocasionada pela DRC piora a qualidade de vida dos pacientes em hemodiálise. Dentre as dificuldades do estudo, questões socioeconômicas, baixa escolaridade e atraso em receber os medicamentos para tratar anemia estiveram em prevalência.

Tabela 2 - Distribuição percentual dos indicadores de qualidade em hemodiálise e meta individual preconizada, HUB, Brasília, 2020. 


\begin{tabular}{|c|c|c|c|c|c|}
\hline INDICADORES & Meta & $\begin{array}{l}<3 \text { meses } \\
\underline{n(7)}\end{array}$ & $\begin{array}{l}>3 e< \\
\text { meses } \\
\underline{n(4)}\end{array}$ & $\begin{array}{l}>6 e< \\
\text { meses } \\
\underline{n(4)}\end{array}$ & $\begin{array}{l}>9 \text { e } 12 \\
\text { meses } \\
\underline{n(13)}\end{array}$ \\
\hline $\begin{array}{l}\text { Proporção de pacientes com Hb } \\
>10 \mathrm{~g} / \mathrm{dl} \text { e }<12,0 \mathrm{~g} / \mathrm{dl} \text { em diálise; }\end{array}$ & $80 \%$ & $33,33 \%$ & $56,25 \%$ & $39,73 \%$ & $50,77 \%$ \\
\hline $\begin{array}{l}\text { Proporção de pacientes em } \\
\text { diálise com Fósforo }(\mathrm{P})>3,5 \mathrm{e}< \\
5,5 \mathrm{mg} / \mathrm{dl} \text {; }\end{array}$ & $50 \%$ & $83,33 \%$ & $75,00 \%$ & $73,81 \%$ & $58,97 \%$ \\
\hline $\begin{array}{l}\text { Proporção de pacientes com } \\
\text { Clearence de ureia } \geq 1.3\end{array}$ & $80 \%$ & $33,33 \%$ & $37,50 \%$ & $50,60 \%$ & $72,82 \%$ \\
\hline $\begin{array}{l}\text { Proporção de pacientes com } \\
\text { valor de PTH > } 600 \mathrm{pg} / \mathrm{ml}\end{array}$ & $<20 \%$ & $0,00 \%$ & $25,00 \%$ & $8,33 \%$ & $26,92 \%$ \\
\hline $\begin{array}{l}\text { Proporção de pacientes com } \\
\text { valores de albumina sérica }<3\end{array}$ & $<20 \%$ & $0,00 \%$ & $0,00 \%$ & $12,50 \%$ & $1,92 \%$ \\
\hline $\begin{array}{l}\text { Proporção de confecção de } \\
\text { Fístula arteriovenosa - FAV } \\
\text { maturadas }\end{array}$ & $70 \%$ & $28,57 \%$ & $37,50 \%$ & $41,96 \%$ & $62,82 \%$ \\
\hline $\begin{array}{l}\text { Incidência de Infecção de acesso } \\
\text { venoso central }\end{array}$ & $<10 \%$ & $7,14 \%$ & $12,50 \%$ & $29,46 \%$ & $1,92 \%$ \\
\hline $\begin{array}{l}\text { Incidência de pacientes com } \\
\text { infecção primária de corrente } \\
\text { sanguínea; }\end{array}$ & $<10 \%$ & $14,29 \%$ & $12,50 \%$ & $11,31 \%$ & $1,92 \%$ \\
\hline $\begin{array}{l}\text { Cateter de curta duração por mais } \\
\text { de } 3 \text { meses; }\end{array}$ & $<10 \%$ & $4,76 \%$ & $14,58 \%$ & $12,50 \%$ & $1,75 \%$ \\
\hline $\begin{array}{l}\text { Taxa de hospitalização dos } \\
\text { pacientes por intercorrência } \\
\text { clínica; }\end{array}$ & $<10 \%$ & $14,29 \%$ & $18,75 \%$ & $62,50 \%$ & $11,54 \%$ \\
\hline
\end{tabular}




\begin{tabular}{l|l|llll}
\hline $\begin{array}{l}\text { Taxa de mortalidade de pacientes } \\
\text { em diálise }\end{array}$ & $<10 \%$ & $0,00 \%$ & $25,00 \%$ & $25,00 \%$ & $0,00 \%$ \\
\hline $\begin{array}{l}\text { Proporção de pacientes dentro de } \\
6 \text { meses após admissão, inscritos }\end{array}$ & $80 \%$ & $57,14 \%$ & $75,00 \%$ & $50,00 \%$ & $53,85 \%$ \\
na cadastro nacional de \\
$\begin{array}{l}\text { transplantes }\end{array}$
\end{tabular}

No estudo de Melo ${ }^{17}$, que tinha como objetivo descrever o perfil epidemiológico dos pacientes com DRC, a anemia foi a principal complicação que surgiu após o diagnóstico da doença, dificultada pela demora da dispensação da medicação de alto custo, principal estratégia de reposição do hormônio eritropoietina.

Um indicador que apresentou valores acima da meta foi o fósforo, ficando assim estabelecido: grupo $1(83,33 \%)$, grupo $2(75 \%)$, grupo $3(73,81 \%)$ e grupo $4(58,97 \%)$. Considerando a meta de 50\% (tabela 2), todos os grupos obtiveram sucesso em controlar o fósforo sérico. No estudo de Veras ${ }^{5}, 51 \%$ da amostra estava entre os valores aceitáveis, 3,5 $5,5 \mathrm{mg} / \mathrm{dl}$ de fósforo sérico - valor ligeiramente maior que o ideal.

O descontrole sérico do fósforo em pacientes com DRC é uma complicação importante associada a consequências clínicas graves, dentre as quais: calcificações vasculares, doenças ósseas e hiperparatiroidismo ${ }^{21}$.

O Clearance de úreia $(\mathrm{Kt} / \mathrm{v})$, avaliado mensalmente por meio de coleta pré e pós de uma amostra de sangue, apresentou valores abaixo do necessário para atingir a meta, os quais: grupo $1(33,33 \%)$, grupo $2(37,50 \%)$, grupo $3(50,60 \%)$ e grupo 4 (72,82\%) (tabela 2). O cenário ideal seria que $80 \%$ (Quadro 2) dos pacientes estivesse com Kt/v > 1,3.

Dois estudos utilizaram o Kt/v para verificar a qualidade de vida dos pacientes em hemodiálise considerando, valor aceitável acima ou igual a 1,2. Obtiveram os seguintes 
resultados: no estudo de $\operatorname{Veras}^{5}, 84 \%$ dos pacientes estava com Kt/v > que 1,2; já no estudo de Pereira e Leite ${ }^{19}$, apenas $59 \%$ da amostra obteve valores superiores a 1,2.

Frequentemente o Kt/v é utilizado como parâmetro de qualidade para diálise, haja vista sua fácil execução, se seguidas as orientações necessárias. Um estudo internacional que cruzou parâmetros laboratoriais e socioclínicos, dos autores Sánchez-González ${ }^{22}$, identificou relação negativa entre Kt/v e os domínios de qualidade de vida - alterações físicas, dor corporal e saúde geral.

Contudo a coleta precisa seguir cuidados no momento da sua realização, se notou a falta de rotinas atualizadas e implementadas nos serviços de diálise no que diz respeito a coleta da ureia pré e pós ${ }^{1,5}$.

O paratormônio (PTH) apresentou não conformidade em dois dos grupos, grupo 2 (25\%) e grupo $4(26,92 \%)$ (Quadro 2), enquanto os dois outros grupos obtiveram valores dentro do desejado (150-600 pg/ml), correspondendo a menos que 20\% (tabela 2) dos resultados. Valores fora da normalidade foram descritos no estudo de Veras ${ }^{5}$, onde $38 \%$ da amostra estava com valores superiores ao desejado.

Aliado ao fósforo, as alterações relativas ao PTH são os maiores responsáveis pelos distúrbios ósseos descritos na DRC: elevações constantes e sustentadas do PTH alteram o metabolismo de reposição de cálcio nos ossos. O hiperparatireoidismo secundário grave é resultado desse descontrole, enquanto os valores aceitáveis encontram-se entre 150-600 pg/ml de $\mathrm{PTH}^{23}$.

A albumina sérica obteve valores dentro da meta em todos os 4 subgrupos do estudo. A DRC leva a uma redução das reservas de gordura e de massa magra corporal. Ainda se buscam métodos eficazes capazes de quantificar essa depleção de forma incessante ${ }^{5}$.

Pereira e Leite ${ }^{19}$, considerando albumina sérica adequada superior a $4 \mathrm{~g} / \mathrm{dl}$, perceberam que $65,1 \%$ da amostra teve valor inferior ao desejado. No estudo de Veras ${ }^{5}$, que considerou 
albumina a 3,5 g/dl, 93\% da amostra teve valores acima do desejado, valores bem expressivos e ideais para os pacientes em HD, haja vista o grande risco de desnutrição.

As recomendações nacionais para hemodiálise preconizam que 70\% (Quadro 2) dos pacientes tenha como acesso a fístula arteriovenosa (FAV). Os quatro grupos do nosso estudo não obtiveram êxito nesse indicador, assim distribuídos: grupo 1 (28,57\%), grupo 2 (37,50\%), grupo $3(41,96 \%)$ e grupo $4(62,82 \%)$ (tabela 2$)$. Veras ${ }^{5}$ levou por base $65 \%$ de pacientes com FAV, e teve amostra de $78 \%$ com esse tipo de acesso. Alta prevalência também presente no estudo de Araújo-Ferreira ${ }^{20}$, 88,2\% de FAV como acesso vascular para hemodiálise. E no estudo DOPPS ${ }^{4}$, realizado em múltiplos centros, $66 \%$ dos pacientes utilizava FAV.

Características dos pacientes tornam o procedimento de alto risco, a exemplo de diabéticos, anêmicos graves, hipoalbuminêmicos quando começaram a HD, além de uso prévio de cateter temporário e idade avançada, quando associados a inexperiência do cirurgião vascular, favorecem negativamente na confecção da FAV ${ }^{24}$.

A respeito das infecções de cateter em diálise, IAVC obteve valores acima do ideal nos grupos 2 e 3,12,50\% e 29,46\% (tabela 2), respectivamente. Quando essa infecção perpassa o óstio e atinge a corrente sanguínea, o termo passa a ser IPCS, também descrito como bacteremia, no qual os grupos 1, 2 e 3 não atingiram a meta, 14,29\%, 11,50\% e 14,21\% (tabela 2), respectivamente.

Os critérios diagnósticos para IAV são pacientes submetidos a hemodiálise com hemocultura negativa ou não colhida e pelo menos um dos seguintes sintomas: saída de secreção purulenta no acesso ou hiperemia, dor e edema local. Já para bacteremia os critérios são uma hemocultura positiva associada a um dos seguintes sintomas: febre $>38^{\circ}$, calafrios, tremores, oligúria e hipotensão ${ }^{25}$. 
Em um estudo desenvolvido nos hospitais públicos do Distrito Federal ${ }^{26}$, houve prevalência de secreção em cateter de duplo lúmen (21,3\%) e infecção/sinais de infecção $(7,8 \%)$

Os elevados índices de infecções em cateter aumentam os números de internações, bem como as tornam mais prolongadas, aumentando custos com saúde, morbidade e mortalidade ${ }^{25}$.

Constantemente os pacientes que necessitam de hemodiálise chegam em situações de urgência, impossibilitando a confecção ou implante de um acesso mais ideal, como a FAV e cateter de longa duração. É grande o número de cateteres temporários, e esses permanecem como acesso por um período de tempo longo, elevando as chances de infecções ${ }^{16,26,37}$.

Os grupos 2 e 3 apresentaram prevalência do uso de CCD superior à meta de 10\% (tabela 2), $14,58 \%$ e $12,50 \%$, respectivamente. Dificuldade financeira, falta de cirurgião vascular, e comorbidades - como o diabetes, idade avançada e falência vascular - foram as dificuldades observadas na pesquisa ${ }^{26}$. Apesar de não ser o mais adequado para os pacientes, os cateteres temporários são comum e trazem benefícios aos pacientes que precisam de tratamento imediato 28.

O total de hospitalizações excedeu o preconizado em todos os 4 grupos, em especial no grupo 3 - 62,50\% (tabela 2) de internações relacionada a complicações clínicas. No entanto, não é possível identificar suas causas, o que pode ter interferido negativamente no valor do indicador. Antunes ${ }^{29}$ avaliou hospitalizações em hemodiálise por um período de 13 meses, identificando 71 ocorrências, das quais 25 (35,2\%) ocorreram por causas cardiovasculares, 23 $(32,4 \%)$ por infecção e $23(32,4 \%)$ por outras causas, levando a 7 óbitos no mesmo período.

Pacientes dialíticos têm risco aumentado de complicações quando sofrem internações devido à debilidade sofrida pelo processo da doença crônica. Não há como interromper o tratamento. A depleção nutricional, por exemplo, é um dos fatores que interferem na rápida 
recuperação desses pacientes, bem como facilita o surgimento de lesões por pressão, sepse e necessidade de suporte ventilatório ${ }^{30}$.

A incidência de óbito foi elevada nos grupos 2 e 3, 25\% em ambos, contudo o total de amostra "n" em cada grupo foi pequena, fato que pode gerar não conformidade com a meta. Ao considerarmos a amostra total do estudo, esse valor estaria dentro do percentual preconizado. O número estimado de mortes no censo de 2017 da SBN foi de 25.817 , gerando uma taxa de mortalidade bruta de $19,9 \%{ }^{16}$.

As políticas que normatizam a hemodiálise no Brasil ${ }^{6,7}$ definem que, após 6 meses de admissão, o paciente deve, caso não haja contraindicação, estar inscrito no cadastro nacional de transplante. A meta é $80 \%$, porém nenhum dos 4 grupos do estudo obteve sucesso nesse indicador. As possíveis hipóteses nesse atraso são: execução dos exames pré-transplante, desinteresse dos pacientes, contraindicações clínicas, transferência para outros centros, recusa e óbitos.

\section{Considerações finais}

Os exames laboratoriais $\mathrm{Hb}$ e paratormônio apresentaram não conformidade expressiva, sendo que o primeiro não obteve sucesso em atingir as metas. Grande número de casos de poliglobulia (>12 $\mathrm{g} / \mathrm{dl}$ ) foram observadas no banco de dados laboratoriais.

Os indicadores Fósforo e Albumina, entretanto, mostraram sucesso no controle sérico, todos os grupos estudados estavam com valores dentro do preconizado. Assistência nutricional, tratamento medicamentoso para controle do fósforo e pacientes orientados foram alguns dos cuidados descritos nas evoluções.

$\mathrm{O}$ indicador Kt/v obteve valores abaixo do esperado. Mensalmente faz-se necessário a coleta de ureia pré e pós, que deve seguir orientações minuciosas para evitar erros nos 
resultados. Nesse momento, a capacitação da equipe, principalmente pelo enfermeiro, faz-se necessária.

As infecções de acesso vasculares, internações e mortalidade foram baixas se compararmos com outros estudos.

A heterogeneidade dos grupos foi um fator limitador ao estudo, além das diferenças clínicas, idades, patologias, tempo de HD, acessos e conhecimento sobre a evolução da doença. Dessa forma, sugere-se o desenvolvimento de outras investigações acerca da temática em outros contextos metodológicos e assistenciais, tais como estudos prospectivos com período observacionais alargados, com grupos mais homogêneos e amostras maiores, aliada à observação direta, por exemplo.

O estudo acerca de indicadores permite visão da qualidade do serviço e oferece oportunidade de intervenções eficazes. O passo inicial para mudança deve ser através da identificação dos problemas, reconhecimento da necessidade de ajustes e criação de plano de intervenções por parte das equipes, colocando-o em prática.

\section{Referências}

1. Meira Fernanda Salazar. Doença renal crônica. Rev. Ciência e Saúde 2017; 10(3): 120.

2. Sociedade Brasileira de Nefrologia. Censo da Sociedade Brasileira de Nefrologia 2017. São Paulo: Sociedade Brasileira de Nefrologia 2017.

3. Alvares $\mathrm{J}$, et al. Fatores associados à qualidade de vida de pacientes em terapia renal substitutiva no Brasil. Ciência \& Saúde Coletiva 2013;18(7): 1903-1910. 
4. Dialysis Outcome Practice Patterns Study (DOPPS). 2012 Annual report of the dialysis outcomes and practice patterns study: hemodialysis data 1997-2011. Arbor Research Collaborative for Health, Ann Arbor, MI. [Internet] 2013.

5. Veras S, et al. Padrões de indicadores clínicos de qualidade em hemodiálise. Journal of Nursing UFPE/Revista de Enfermagem UFPE 2016; 10(7): 2641-49.

6. Brasil, Ministério da Saúde. Portaria MS/GM no 389 de 13 de março de 2014. Define os critérios para a organização da linha de cuidado da Pessoa com Doença Renal Crônica (DRC) e institui incentivo financeiro de custeio destinado ao cuidado ambulatorial prédialítico. Diário Oficial [da] República Federativa do Brasil, Brasília 2014.

7. Brasil. Ministério da Saúde. Portaria n ${ }^{\circ} 1.675$, de 7 de junho de 2018. Altera a Portaria de Consolidação nº 3/GM/MS, de 28 de setembro de 2017, e a Portaria de Consolidação no 6/GM/MS, de 28 de setembro de 2017, para dispor sobre os critérios para a organização, funcionamento e financiamento do cuidado da pessoa com Doença Renal Crônica - DRC no âmbito do Sistema Único de Saúde - SUS. Diário Oficial [da] República Federativa do Brasil, Brasília 2018; p 1-21.

8. Brasil. Ministério da Saúde. Agência Nacional de Vigilância Sanitária. Resolução RDC no 11, de 13 de março de 2014. Dispõe sobre os requisitos de boas práticas de funcionamento para os serviços de diálise e dá outras providências. Diário Oficial [da] República Federativa do Brasil, Brasília 2014.

9. Grangé S, et al. Monitoring of hemodialysis quality-of-care indicators: why is it important?. BMC nephrology 2013. 14(1): 109. 
10. Fernandes MICD, Andrade Silva PK, Medeiros Danta, AL., Paiva MGMN, Azevedo Araújo MG, Carvalho Lira ALB. Pacientes em hemodiálise com diagnóstico de enfermagem volume de líquidos excessivo: aspectos socioeconômicos e clínicos. Cogitare Enfermagem 2015; 20(1).

11. Aragão J. Introdução aos estudos quantitativos utilizados em pesquisas científicas. Revista práxis $2013 ; 3(6)$.

12. Gil AC. Como elaborar projetos de pesquisa. Ed. Atlas. 2010; $5^{\circ}$ edição.

13. Gerhardt TE, Silveira DT. Métodos de pesquisa. Ed. Plageder 2009.

14. Álvares AM, Lobato, GR. Um estudo exploratório da incidência de sintomas depressivos em crianças e adolescentes em acolhimento institucional. Temas em Psicologia 2013; 21(1): 151-164.

15. Brasil, Conselho Nacional de Saúde. Resolução 466/2012-Normas para pesquisa envolvendo seres humanos. Diário Oficial [da] República Federativa do Brasil, Brasília 2012.

16. Thomé FS, et al. Inquérito Brasileiro de Diálise Crônica 2017. J. Bras. Nefrol. [Internet] 2019; 41 (2): 208-214.

17. Melo WF, Bezerra ALD, Sousa MNA. Perfil epidemiológico de pacientes com insuficiência renal crônica: um estudo quantitativo. Rev eletrônica Fainor [Internet] 2014; 7 (2): $142-56$. 
18. Nerbass FB, et al. Diferenças na fosfatemia e na frequência de consumo de fontes alimentares de fósforo em pacientes em hemodiálise do sul e norte do Brasil. J. Bras. Nefrol. [Internet] 2019; 41 (1): 83-88.

19. Pereira CV, Leite ICG. Qualidade de vida relacionada à saúde de pacientes em terapêutica hemodialítica. Acta paul. enferm. [Internet] 2019; 32(3): 267-274.

20. Araújo-Ferreira, et al. Conhecimento: processo da doença em pacientes submetidos à hemodiálise. Investigación y Educación en Enfermería 2018; 36(2).

21. Gesualdo GD, et al. Fatores associados à qualidade de vida de pacientes em hemodiálise. Texto contexto - enferm [Internet] 2017; 26(2): e05600015.

22. Sánchez-González JC, et al. Análisis de la calidad de vida en pacientes con tratamiento renal sustitutivo: influencia de los parámetros analíticos y socioclínicos. Enferm Nefrol 2019; 22(2):159-67.

23. Bucharles SGE, et al. Impacto de cinacalcete em marcadores do metabolismo mineral de pacientes em diálise portadores de hiperparatireoidismo secundário grave. Brazilian Journal of Nephrology 2019; (AHEAD). 41( 3 ): 336-344.

24. Siga EL, Ibalo N, Benegas MR, Laura F, Luna C, Aiziczon DH, et al. Relevância de um cirurgião vascular qualificado e práticas hospitalares otimizadas na patência a longo prazo das fístulas arteriovenosas: um estudo prospectivo. Brazilian Journal of Nephrology 2019; 41(3): 330-335. 
25. Brasil. Nota técnica GVIMS/GGTES/ANVISA: Sistema Nacional de Vigilância Epidemiológica das Infecções Relacionadas à Assistência à Saúde em Serviços de Diálise, 2019.

26. Rocha RPF, Pinho DLM. Ocorrência de eventos adversos em unidades públicas de hemodiálise. Enferm. glob. [Internet] 2019; 18 (55): 1-34.

27. Clementino DC, et al. Pacientes em hemodiálise: importância do autocuidado com a fístula arteriovenosa. Rev enferm UFPE on line 2018; 12(7): 1841-52.

28. Schwanke AA, et al. Central venous catheter for hemodialysis: incidence of infection and risk factors. Rev Bras Enferm [Internet] 2018; 71(3):1115-21.

29. Antunes SA, Canziani MEF, Campos AF, Vilela RQB. A hipoalbuminemia parece estar associada a uma maior taxa de hospitalização nos pacientes em hemodiálise. J. Bras. Nefro. 2016; 38(1): 70-75.

30. Aguiar FP, et al. Características e preditores de doença crítica crônica na unidade de terapia intensiva. Revista Brasileira de Terapia Intensiva 2019; 31(4): 511-20. 